\title{
475786 - CASE REPORT - TYPE A AORTIC DISSECTION IN A PARTURIENT: USE OF 3-D TRANSESOPHAGEAL ECHOCARDIOGRAPHY \& FACTOR VIIA IN A COMBINED PROCEDURE
}

\author{
May-Sann Yee, MD, Philip Waters, M.D.C.M \\ Anesthesia \& Pain Medicine, University of Alberta Hospitals, Edmonton, AB, \\ Canada
}

Purpose: Acute aortic dissection during third-trimester pregnancy represents an uncommon complication with high risk to both mother and fetus. No consensus has yet been reached on the management of complex, interdependent issues regarding surgical treatment of the dissection, timing of delivery, anticoagulation, or cardiopulmonary bypass.

Clinical Features: Signed consent was obtained from the patient prior to commencement, with the understanding that no identifying data would be included. Our case report is of a 36 year-old female presenting at 35 weeks gestation with a Type A aortic dissection and pericardial effusion. Given her tenuous hemodynamic status, and remote chance of tolerating a Cesarean section prior to aortic repair, the approach was to do a combined procedure. Following induction of general anesthesia with cardiovascular surgery and obstetric standby, a median sternotomy was performed, the pericardium was opened, and cardiopulmonary bypass sites were exposed. Immediately following, the obstetricians performed a low segment transverse Cesarean section and the baby was delivered.

Conclusion: We will review the multidisciplinary discussion regarding the planning of our intervention, the novel use of three-dimensional echocardiography in precisely defining the extent of the aortic and innominate artery dissection, and the management of uterine and thoracic bleeding using Factor VIIa.

References: 1 . ImmerR et al. Aortic dissection in pregnancy: analysis of risk factors and outcomes. Ann Thorac Surg. 2003 Jul;76(1):309-14 2. Pinosky ML et al. Anesthesia for simultaneous cesarean section and acute aortic dissection repair in a patient with Marfan's syndrome. J Cardiothorac Vasc Anesth. 1994 Aug;8(4): 451-4 3. Ravaoherisoa J et al. (Dissecting ascending aortic aneurysm in pregnancy: simultaneous cesarean section and surgical aortic repair). Ann Fr Anesth Reanum. 1997;16(8):974-77 Sakaguchi M et al. Surgery for acute Type A aortic dissection in pregnant patiens with Marfan syndrome. Eur J Cardiothorac Surg. 2005 (28): 280-85 5. Weissman-Brenner A et al. Aortic dissection in pregnancy. Obstet Gyne. 2004. May;103(5):S1110-3 